\title{
Generalized convergence conditions of the parameter adaptation algorithm in discrete-time recursive identification and adaptive control
}

\author{
Bernard Vau ${ }^{\text {a }}$, Henri Bourlès ${ }^{\mathrm{a}}$ \\ ${ }^{a}$ SATIE Ecole normale supérieure de Paris-Saclay, 94230 CACHAN France.
}

\begin{abstract}
In this paper, we extend convergence conditions for the parameter adaptation algorithm, used in discrete-time recursive identification schemes, or in adaptive control. Whereas the classical stability analysis of this algorithm consists in checking the strictly real positiveness of an associated transfer function, we demonstrate that convergence can be obtained even when this condition is not fulfilled, under some assumptions on the algorithm forgetting factors. These results regarding both deterministic and stochastic contexts are obtained by analyzing convergence with a prescribed degree of stability.
\end{abstract}

Key words: Discrete-time recursive identification; adaptive control; parameters estimation.

\section{Introduction}

The parameter adaptation algorithm (PAA) described in [Landau, Lozano, M' Saad, Karimi, 2011] is a cornerstone in adaptive control. It provides an on-line parameter estimation of a discrete-time system and is extensively used in recursive identification schemes. The issue of PAA convergence analysis has been addressed for a long time [Landau, 1965] by considering that this algorithm can always be represented as an equivalent closed-loop including a linear time-varying (LTV) feedback system in interaction with a discrete feedforward linear time-invariant (LTI) system. Hyperstability theory imposes the strictly real positiveness condition of the transfer function linked to the feedforward LTI system and, as this condition is only sufficient, in some cases when it is not fulfilled, the PAA convergence can be nevertheless observed. The purpose of this paper is to provide less restrictive convergence conditions for the PAA. We show that under classical assumptions on the algorithm forgetting factors, such as those considered in, e.g., [Lozano, 1983], the algorithm stability can be proved even if the LTI system strictly real positiveness is not satisfied. These results provide new analysis tools able to cope, in particular, with an LTI system transfer function having poles on the unit circle. The here-

Email addresses: bernard.vau@satie.ens-cachan.fr (Bernard Vau), henri.bourles@satie.ens-cachan.fr (Henri Bourlès). after developments are based upon controlled LTI systems with a prescribed degree of stability that have been studied in [Anderson, Moore, 1971] and [Bourlès, 1987] in the continuous-time case, and in [Bourlès, 1990] in the discrete-time-case. Likewise Kalman filters with a prescribed degree of stability have been developed in [Anderson, Moore 1979]. Since the PAA is a variant of the Kalman filter, we combine these approaches in the sequel with developments achieved in a deterministic context [Landau, Silveira, 1979] and in a stochastic context [Landau, 1982] to obtain these generalized convergence conditions.

\section{Deterministic context}

In the beginning of this section, we refer systematically to [Landau, Lozano, M'Saad, Karimi, 2011] (pp.102103) for the description of the PAA, and in particular we reuse the same notation. The PAA aims at making as close to zero as possible a prediction (or adaptation) error between the output of the system to be identified and an adjustable model output. Let us denote by: $\nu(t+1)$ : The a-posteriori adaptation error (scalar), $\phi(t)$ : The observation vector $\left(\right.$ size $\left.\left(n_{\phi}, 1\right)\right)$,

$\theta$ : The parameters vector to be estimated $\left(\right.$ size $\left.\left(n_{\phi}, 1\right)\right)$, $\widehat{\theta}(t)$ : The current estimated parameters vector (size $\left.\left(n_{\phi}, 1\right)\right)$.

We consider systems for which the a-posteriori error is 
given by:

$$
\nu(t+1)=H\left(q^{-1}\right)(\theta-\widehat{\theta}(t+1))^{T} \phi(t)
$$

In this expression $H\left(q^{-1}\right)$ is the operator associated with the (bicausal) transfer function $H\left(z^{-1}\right)$, which is a ratio of two monic polynomials. The PAA equations are:

$$
\begin{gathered}
\widehat{\theta}(t+1)=\widehat{\theta}(t)+F(t) \phi(t) \varepsilon(t+1) \\
F^{-1}(t+1)=\lambda_{1} F^{-1}(t)+\lambda_{2} \phi(t) \phi^{T}(t)
\end{gathered}
$$

$F(t)$ is the adaptation gain (positive definite matrix), $0<\lambda_{1} \leq 1,0 \leq \lambda_{2}<2$ are the forgetting factors. A sufficient convergence condition for the PAA, is that the transfer function:

$$
H\left(z^{-1}\right)-\frac{\lambda_{2}}{2}
$$

be strictly positive real. From equations (1),(2),(3) an equivalent closed-loop can be drawn. For this purpose let us denote by: $\tilde{\theta}(t)=\widehat{\theta}(t)-\theta$ : the parameters vector error $\left(\operatorname{size}\left(n_{\phi}, 1\right)\right)$,

$u(t+1)=-\phi^{T}(t) \tilde{\theta}(t+1)$ : the input of $H\left(q^{-1}\right)$ (scalar), $y(t+1)=\nu(t+1)$ : the output of $H\left(q^{-1}\right)$ (scalar),

$\tilde{u}(t+1)=\nu(t+1)$ : the associated LTV system input, $\tilde{y}(t+1)=\phi^{T}(t) \tilde{\theta}(t+1)$ : the LTV system output (scalar), $\tilde{x}(t)=\tilde{\theta}(t)$ : the LTV system state vector $\left(\right.$ size $\left.\left(n_{\phi}, 1\right)\right)$, $x(t)$ : the state vector of the operator $H\left(q^{-1}\right)$ (size $(n, 1)$ ). The LTV system state space equations are:

$$
\begin{aligned}
& \tilde{x}(t+1)=\tilde{A}(t) \tilde{x}(t)+\tilde{B}(t) \tilde{u}(t+1) \\
& \tilde{y}(t+1)=\tilde{C}(t) \tilde{x}(t)+\tilde{D}(t) \tilde{u}(t+1)
\end{aligned}
$$

With:

$\tilde{A}(t)=I_{n_{\phi}}$,

$\tilde{B}(t)=F(t) \phi(t),\left(\right.$ size $\left.\left(n_{\phi}, 1\right)\right)$,

$\tilde{C}(t)=\phi^{T}(t),\left(\right.$ size $\left.\left(1, n_{\phi}\right)\right)$,

$\tilde{D}(t)=\phi^{T}(t) F(t) \phi(t)$, (scalar).

In what follows, a controllable and observable linear system is considered as an operator associating the output to the input, with zero initial conditions. The LTI system state space equations are written:

$$
\begin{aligned}
x(t+1) & =A x(t)+B u(t) \\
y(t) & =C x(t)+D u(t)
\end{aligned}
$$

With $A$ of size $(n, n)$,

$B$ of size $(n, 1)$,

$C$ of size $(1, n)$

$D$ a scalar.

The equivalent closed-loop is represented in Fig.1. For any signal $s=\{s(t)\}$ (determinist or stochastic), denote by $s_{\rho}$ the signal $\left\{\rho^{t} s(t)\right\}, \rho \geq 1$. Considering the signal

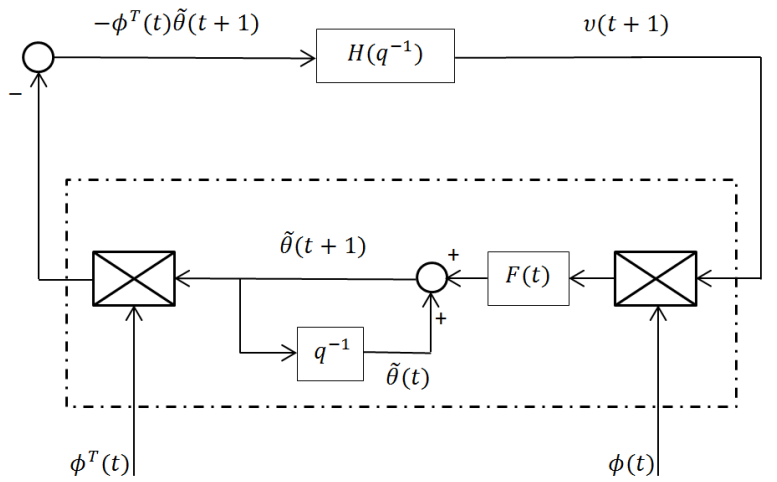

Fig. 1. Classical PAA closed-loop

$y(t)=H\left(q^{-1}\right) u(t)$, the relation between $y_{\rho}(t)$ and $u_{\rho}(t)$ is $y_{\rho}(t)=H_{\rho}\left(q^{-1}\right) u_{\rho}(t)$, with $H_{\rho}\left(q^{-1}\right)=H\left(\rho q^{-1}\right)$, [Bourlès, 1990]. An equivalent closed-loop can be derived from the loop represented in Fig.1, in which the feedback LTV system input and output are now $\tilde{u}_{\rho}(t+1)$, $\tilde{y}_{\rho}(t+1)$, and the feedforward LTI system input and output correspond to $u_{\rho}(t)$ and $y_{\rho}(t)$. The state space equations of the so-called $\rho$-LTV system are given by:

$$
\begin{aligned}
& \tilde{x}_{\rho}(t+1)=\tilde{A}_{\rho}(t) \tilde{x}_{\rho}(t)+\tilde{B}_{\rho}(t) \tilde{u}_{\rho}(t+1) \\
& \tilde{y}_{\rho}(t+1)=\tilde{C}_{\rho}(t) \tilde{x} \rho(t)+\tilde{D}_{\rho}(t) \tilde{u}_{\rho}(t+1)
\end{aligned}
$$

With:

$\tilde{A}_{\rho}=\rho I_{n_{\phi}}$,
$\tilde{B}_{\rho}=F(t) \phi(t)\left(\right.$ size $\left.\left(n_{\phi}, 1\right)\right)$,
$\tilde{C}_{\rho}(t)=\rho \phi^{T}(t)\left(\right.$ size $\left.\left(1, n_{\phi}\right)\right)$,
$\tilde{D}_{\rho}=\phi^{T}(t) F(t) \phi(t)$ (scalar).

Fig. 2 describes the equivalent PAA closed-loop, with $\rho$-signals.

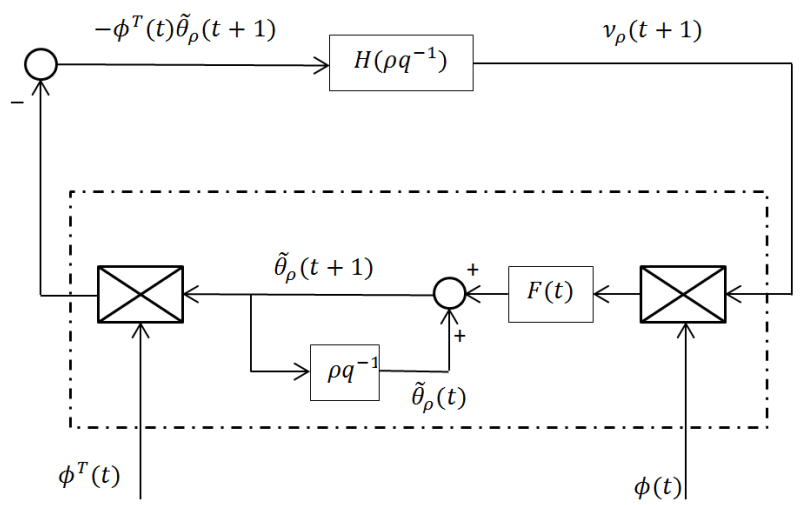

Fig. 2. Equivalent PAA closed-loop with $\rho$-signals

Imposing that $\tilde{y}_{\rho}(t)$ and $\tilde{u}_{\rho}(t)$ converge towards 0 is 
equivalent to impose a degree of stability $\rho$ to the classical closed-loop in Fig. 1.

Theorem 1 Consider the PAA algorithm given by (1), (2), and (3). Assume that there exists $\rho \geq 1$ such that the following conditions hold:

(1) $\lambda_{1} \leq 2-\rho^{2}, 0 \leq \lambda_{2}<2$

(2) The transfer function $H\left(\rho z^{-1}\right)-\frac{\lambda_{2}}{2}$ is strictly positive real.

Then one has:

$$
\begin{gathered}
\lim _{t \rightarrow \infty} \nu_{\rho}(t+1)=0 \\
\lim _{t \rightarrow \infty}[\theta-\widehat{\theta}(t+1)]^{T} \phi(t) \rho^{t}=0 \\
\lim _{t \rightarrow \infty}[\rho(\theta-\widehat{\theta}(t+1))-(\theta-\widehat{\theta}(t))]^{T} F^{-1}(t) \cdots \\
\cdots[\rho(\theta-\widehat{\theta}(t+1))-(\theta-\widehat{\theta}(t))] \rho^{2 t}=0 \\
{[\widehat{\theta}(t)-\theta]^{T} F^{-1}(t)[\widehat{\theta}(t)-\theta] \rho^{2 t}<\text { const }<\infty}
\end{gathered}
$$

Proof: Since the case $\rho=\lambda_{1}=1$ has already been treated in ([Landau, Lozano, M' Saad, Karimi, 2011], Thm. 3.1) we assume $\rho>1$. The transfer function $H\left(\rho z^{-1}\right)-\frac{\lambda_{2}}{2}$ is strictly real positive if and only if (by definition) $H\left(\rho z^{-1}\right)$ belongs to the class $L(\Lambda)$, as defined in [Landau, Lozano, M' Saad, Karimi, 2011], p. 556. The feedback loop using $\rho$-signals is stable and $\tilde{y}_{\rho}(t), \tilde{u}_{\rho}(t)$ converge towards 0 if the LTV system (6), belongs to the class $N(\Gamma)$, as defined in [Landau, Lozano, M' Saad, Karimi, 2011], p. 558, with $\Gamma=\lambda_{2}$. According to lemma $C .7$ of the same reference, the system (6) belongs to the class $N(\Gamma)$ if there exist three sequences of non-negative definite symmetric matrices $\{P(t)\},\{R(t)\},\{Q(t)\}$ and a matrix sequence $\{S(t)\}$ such that:

$$
\begin{aligned}
& \tilde{A}_{\rho}^{T}(t) P(t+1) \tilde{A}_{\rho}(t)-P(t)=-Q(t)+\tilde{C}_{\rho}^{T}(t) \Gamma \tilde{C}_{\rho}(t) \\
& \tilde{C}_{\rho}(t)-\tilde{B}_{\rho}^{T}(t) P(t+1) \tilde{A}_{\rho}(t)=S^{T}(t)-\tilde{D}_{\rho}^{T}(t) \Gamma \tilde{C}_{\rho}(t)
\end{aligned}
$$

$\tilde{D}_{\rho}(t)+\tilde{D}_{\rho}^{T}(t)-\tilde{B}_{\rho}^{T}(t) P(t+1) \tilde{B}_{\rho}(t)=R_{\rho}(t)-\tilde{D}_{\rho}^{T}(t) \Gamma \tilde{D}_{\rho}(t)$

$$
\left[\begin{array}{ll}
Q(t) & S(t) \\
S^{T}(t) & R(t)
\end{array}\right] \geq 0
$$

By choosing $P(t+1)=F^{-1}(t+1)$, (11) leads to $Q(t)=\left(1-\lambda_{1} \rho^{2}\right) F^{-1}(t)$ and $\Gamma=\lambda_{2}$ (since $\tilde{A}_{\rho}=\rho I_{n_{\phi}}$ and $\left.\tilde{C}_{\rho}(t)=\rho \phi^{T}(t)\right) ;(12)$ yields $S(t)=\rho\left(1-\lambda_{1}\right) \phi^{T}(t)$, (since $\tilde{B}_{\rho}=F(t) \phi(t)$ and $\tilde{D}_{\rho}=\phi^{T}(t) F(t) \phi(t)$ ) and with (13) we get $R(t)=\left(2-\lambda_{1}\right) \phi^{T} F(t) \phi(t)$. Using the Schur complement, and assuming $\lambda_{1}<1 / \rho^{2}$ (in order that $Q(t)$ be non negative), the condition (14) is equivalent to $R(t)-S^{T}(t) Q^{-1}(t) S(t) \geq 0$, which is equivalent to $\left(2-\lambda_{1}\right)-\rho^{2}\left(1-\lambda_{1}\right)^{2} /\left(1-\lambda_{1} \rho^{2}\right) \geq 0$ i.e. $\lambda_{1} \leq 2-\rho^{2}$. Notice that $2-\rho^{2}<1 / \rho^{2}$ since $\rho>1$. Thus according to theorem C.1 of [Landau, Lozano, M'Saad, Karimi, 2011], we obtain:

$$
\begin{gathered}
\lim _{t \rightarrow \infty} y_{\rho}(t+1)=\lim _{t \rightarrow \infty} u_{\rho}(t+1)=\lim _{t \rightarrow \infty} \nu_{\rho}(t+1)=0 \\
\tilde{x}_{\rho}^{T}(t) F^{-1}(t) \tilde{x}_{\rho}(t) \leq \mathrm{const}<\infty \\
\lim _{t \rightarrow \infty}\left[\begin{array}{cr}
\tilde{x}_{\rho}^{T}(t) & \left.\tilde{u}_{\rho}^{T}(t+1)\right]\left[\begin{array}{cc}
Q(t) & S(t) \\
S^{T}(t) & R(t)
\end{array}\right]\left[\begin{array}{c}
\tilde{x}_{\rho}(t) \\
\tilde{u}_{\rho}(t+1)
\end{array}\right]=0 \\
\lim _{t \rightarrow \infty} \tilde{x}_{\rho}^{T}(t) F^{-1}(t) \tilde{x}_{\rho}(t)=\mathrm{const}
\end{array}\right.
\end{gathered}
$$

The interest of theorem 1 is that it provides a tool to assess the PAA stability even if the transfer function $H\left(z^{-1}\right)-\frac{\lambda_{2}}{2}$ is not strictly real positive. For $\rho>1$, convergence is obtained with a stability degree depending on $\rho$. The price to pay lies in the fact that if $\rho>1, \lambda_{1}$ must be strictly inferior to 1 , and therefore the adaptation gain $F(t)$ cannot tend towards 0 .

\section{Stochastic context}

Let us assume now that the system is disturbed by an output noise $\omega(t+1)$. Then the a-posteriori adaptation error is given by:

$$
\nu(t+1)=H\left(q^{-1}\right)(\theta-\widehat{\theta}(t+1))^{T} \phi(t)+\omega(t+1)
$$

$\omega(t+1)$ is a martingale adapted to the sequence of $\sigma$-algebra $\mathcal{F}_{t}$ where $\mathcal{F}_{t}$ is generated by the observation up to and including t. Additionally the following conditions are assumed to hold; they are similar to those of [Landau, Lozano, M' Saad, Karimi, 2011], and [Landau, 1982] :

$$
\begin{gathered}
\mathbf{E}\left[\omega(t+1) \mid \mathcal{F}_{t}\right]=0 \\
\mathbf{E}\left[\omega^{2}(t+1) \mid \mathcal{F}_{t}\right]=\sigma^{2}<\infty \\
\lim _{t \rightarrow \infty} \frac{1}{N} \sum_{t=0}^{N} \omega^{2}(t)<\infty
\end{gathered}
$$

We will prove the following lemmas and theorem: 
Lemma 1 Assume that there exists $\delta>0$ such that for any $t \geq n_{\phi}, \sum_{i=t+1}^{t+n_{\phi}} \phi(i) \phi^{T}(i)-\delta I$ is positive definite. Then one has for any $\epsilon>0$ :

$$
\sum_{t=1}^{\infty} \frac{\operatorname{trace}(F(t+1))}{(t+1)^{\epsilon}}<\infty
$$

Proof: The proof is essentially based on theorem 1 of [Lozano, 1983]. Denote by $\underline{\sigma}($.$) and \bar{\sigma}($.$) the smallest and$ largest singular value, respectively. According to this reference one has:

$F^{-1}(t+1)=\lambda_{1}^{t+1} F^{-1}(0)+\lambda_{2} \sum_{i=0}^{t} \lambda_{1}^{t-i} \phi(i) \phi^{T}(i)$, thus: $\underline{\sigma}\left(F^{-1}(t+1)\right) \geq \lambda_{2} \underline{\sigma}\left(\sum_{i=0}^{t} \lambda_{1}^{t-i} \phi(i) \phi^{T}(i)\right)$

$\geq \lambda_{2} \underline{\sigma}\left(\sum_{i=t-n_{\phi}+1}^{t} \lambda_{1}^{t-i} \phi(i) \phi^{T}(i)\right)$, and: $\underline{\sigma}\left(F^{-1}(t+1)\right) \geq \lambda_{2} \lambda_{1}^{n_{\phi}} \underline{\sigma}\left(\sum_{i=t-n_{\phi}+1}^{t} \phi(i) \phi(i)^{T}\right)$.

The assumption yields: $\underline{\sigma}\left(F^{-1}(t+1)\right) \geq \lambda_{2} \lambda_{1}^{n_{\phi}} \delta$. Therefore we have $\bar{\sigma}\left(F(t+1) \leq 1 /\left(\lambda_{2} \lambda_{1}^{n_{\phi}} \delta\right)<\infty\right.$. Since $F(t+1)$ is a symmetric definite positive matrix, the eigenvalues of $F(t+1)$ coincide with its singular values. Thus we have: $\operatorname{trace}(F+1) \leq n_{\phi} /\left(\lambda_{2} \lambda_{1}^{n_{\phi}} \delta\right)<\infty$, and we obtain $\sum_{t=1}^{\infty} \frac{\operatorname{trace}(F(t+1))}{(t+1)^{\epsilon}}<\infty$, as desired.

Lemma 2 For $\lambda_{1} \leq 1$ and $\epsilon>0$ one has:

$$
\sum_{t=1}^{\infty} \frac{\phi^{T}(t) F^{2}(t+1) \phi(t)}{(t+1)^{\epsilon}}<\infty
$$

Proof: The case $\lambda_{1}=1$ has been treated by [Landau, 1982] and [Landau, Lozano, M' Saad, Karimi, 2011] (pp. 142143), so it remains to check the case $\lambda_{1}<1$. For this purpose, we notice at once that:

$\phi^{T}(t) F^{2}(t+1) \phi(t) \leq \frac{1}{\lambda_{1}} \phi^{T}(t) F(t) F(t+1) \phi(t)$. This result is obtained from the inversion lemma applied to (3), yielding: $F(t+1)=\frac{1}{\lambda_{1}}\left[F(t)-\frac{F(t) \phi(t) \phi(t)^{T} F(t)}{\frac{\lambda_{1}}{\lambda_{2}}+\phi(t)^{T}(t) F(t) \phi(t)}\right]$ and by left-and right-multiplying by $F(t+1)$, pre-multiplying by $\phi(t)^{T}$ and post-multiplying by $\phi(t)$. We can write: $\phi^{T}(t) F^{2}(t+1) \phi(t) \leq \frac{1}{\lambda_{1}} \operatorname{trace}\left(\phi^{T}(t) \phi(t) F(t+1) F(t)\right)$, which is equivalent -because of $(3)$ - to $\phi^{T}(t) F^{2}(t+$ 1) $\phi(t) \leq \frac{1}{\lambda_{1} \lambda_{2}} \operatorname{trace}\left(\left(F^{-1}(t+1)-\lambda_{1} F^{-1}(t)\right) F(t+1) F(t)\right)$, yielding:

$\phi^{T}(t) F^{2}(t+1) \phi(t) \leq$

$\frac{1}{\lambda_{2} \lambda_{1}} \operatorname{trace}(F(t)-F(t+1))+\frac{1-\lambda_{1}}{\lambda_{2} \lambda_{1}} \operatorname{trace}(F(t+1))$

By induction we obtain:

$\sum_{t=1}^{\infty} \frac{\left(\phi^{T}(t) F^{2}(t+1) \phi(t)\right)}{(t+1)^{\epsilon}} \leq$

$\frac{1}{\lambda_{2} \lambda_{1}} \operatorname{trace}(F(0))+\frac{\left(1-\lambda_{1}\right)}{\lambda_{2} \lambda_{1}} \sum_{t=1}^{\infty} \frac{\operatorname{trace}(F(t+1))}{(t+1)^{\epsilon}}$,

and by Lemma 1 we get:

$\sum_{t=1}^{\infty} \frac{\phi^{T}(t) F^{2}(t+1) \phi(t)}{(t+1)^{\epsilon}}<\infty$.
Theorem 2 Let us assume that there exist $\rho>1$ for which the following conditions hold:

(1) $\lambda_{1} \leq 2-\rho^{2}$

(2) $H\left(\rho z^{-1}\right)-\frac{\lambda_{2}}{2}$ is strictly positive real,

(3) $\lim _{N \rightarrow \infty} \frac{1}{N} \sum_{t=0}^{N} \phi(i)^{T} \phi(i)<\infty$,

(4) There exists $\delta>0$ such that for any $t \geq n_{\phi}$, $\sum_{i=t+1}^{t+n_{\phi}} \phi(i)^{T} \phi(i)-\delta I$ is positive definite, where $n_{\phi}$ is the length of vector $\phi(t)$.

Then, for any $\epsilon>0$, we have:

$$
\lim _{N \rightarrow \infty} \frac{1}{N^{(1+\epsilon)}} \sum_{t=1}^{N}[\nu(t)-\omega(t)]^{2}=0 \quad \text { a.s. }
$$

$\lim _{N \rightarrow \infty} \frac{1}{N^{(1+\epsilon)}} \sum_{t=1}^{N} \nu^{2}(t)=\lim _{N \rightarrow \infty} \frac{1}{N^{(1+\epsilon)}} \sum_{t=1}^{N} \omega^{2}(t)$

$$
\lim _{N \rightarrow \infty} \frac{1}{N^{(1+\epsilon)}} \sum_{t=1}^{N}\left[(\widehat{\theta}(t)-\theta)^{T} \phi(t-1)\right]^{2}=0
$$

Proof: The proof is based on [Landau, 1982], and on [Landau, Lozano, M'Saad, Karimi, 2011] (theorem D.3). Set $\bar{y}(t+1)=\theta^{T}(t+1) \phi(t)$; According to the second reference (pp. 141-142) one has: $\mathbf{E}\left[\bar{y}(t+1) \omega(t+1) \mid \mathcal{F}_{t}\right]=\gamma(t) \phi^{T}(t) F(t+1) \phi(t) \sigma^{2}$ with $\gamma(t)=\frac{\left(\lambda_{1}+\lambda_{2} \phi^{T}(t) F(t) \phi(t)\right)}{\left(1+\phi^{T}(t) F(t) \phi(t)\right)}$. This expression of $\gamma(t)$ is obtained from the expressions (4.104) and (4.106) of the same book, and from the inversion matrix lemma applied to (3). Let $r(t+1)=t^{(1+\epsilon)} \rho^{2 t}$ and: $\alpha(t+1)=$ $\mathbf{E}\left[\frac{\left(\bar{y}_{\rho}(t+1) \omega_{\rho}(t+1)\right)}{r(t+1)} \mid \mathcal{F}_{t}\right]=\mathbf{E}\left[\frac{(\bar{y}(t+1) \omega(t+1))}{(t+1)^{(1+\epsilon)}} \mid \mathcal{F}_{t}\right]$.

One can check that in the case $\lambda_{1}<1$, and $0<\gamma(t)<2$, conditions of theorem D.3 mentioned above are:

a) $\Lambda-\Gamma(t) \geq 0$, which is true since 1) and 2) are satisfied simultaneously,

b) $r(t+1) \geq r(t)$ which is immediate for $\rho>1$ and $\epsilon>0$

c) $\alpha(t+1) \geq 0$ equivalent to $\gamma(t) \phi^{T}(t) F(t+1) \phi(t) \sigma^{2} \geq$ 0 . That is true because $F(t+1)$ is a positive definite matrix and $\gamma(t)>0, \sigma^{2} \geq 0$.

d) $\sum_{t=1}^{\infty} \alpha(t)<\infty$.

In order to satisfy d), since $\gamma(t)>0$, we must verify that: $\sum_{t=1}^{\infty} \frac{\phi^{T}(t) F(t+1) \phi(t)}{(t+1)^{(1+\epsilon)}}<\infty$. According to lemma 2, we have $\sum_{t=1}^{\infty} \frac{\phi^{T}(t) F^{2}(t+1) \phi(t)}{(t+1)^{\epsilon}}<\infty$

Besides, we get: $\phi^{T}(t) F(t+1) \phi(t)=$ $\phi^{T}(t) F(t+1) F^{-1}(t+1) F(t+1) \phi(t)$. Then: 
$\phi^{T}(t) F(t+1) \phi(t) \leq$

trace $\left(F^{-1}(t+1) \phi^{\bar{T}}(t) F^{2}(t+1) \phi(t)\right)$, and owing to $(2)$

$\left.\operatorname{trace}\left(F^{-1}(t+1)\right) \leq \operatorname{trace}\left(F^{-1}(t)\right)+\lambda_{2} \phi^{T}(t) \phi(t)\right)$.

By induction we obtain:

$\operatorname{trace}\left(F^{-1}(t+1)\right) \leq \lambda_{2} \sum_{i=0}^{t} \phi^{T}(t) \phi(t)+\operatorname{trace}\left(F^{-1}(0)\right)$

and condition (3) of the theorem leads to:

$\sup _{t \geq 1} \frac{\operatorname{trace}\left(F^{-1}(t+1)\right)}{(t+1)}<\infty$. Therefore we get:

$\sum_{t=1}^{\infty} \frac{\phi^{T}(t) F(t+1) \phi(t)}{(t+1)^{(1+\epsilon)}} \leq$

$\sum_{t=1}^{\infty} \frac{\operatorname{trace}\left(F^{-1}(t+1)\right)}{(t+1)} \frac{\phi^{T}(t) F^{2}(t+1) \phi(t)}{(t+1)^{\epsilon}} \leq$

$\leq \sup _{t \geq 1}\left(\frac{\operatorname{trace}\left(F^{-1}(t+1)\right)}{(t+1)}\right) \sum_{t=1}^{\infty} \frac{\phi^{T}(t) F^{2}(t+1) \phi(t)}{(t+1)^{\epsilon}}$

and since $\sum_{t=1}^{\infty} \frac{\phi^{T}(t) F^{2}(t+1) \phi(t)}{(t+1)^{\epsilon}}<\infty$ by lemma 2, we finally obtain:

$\sum_{t=1}^{\infty} \alpha(t)=\sum_{t=1}^{\infty} \frac{\phi^{T}(t) F(t+1) \phi(t)}{(t+1)^{(1+\epsilon)}}<\infty$, meaning that the condition d) is satisfied. This ends the proof.

As $\lambda_{1}$ is necessarily strictly inferior to 1 , in the stochastic context the vector $\widehat{\theta}(t)$ does not converge towards a constant value. However, as shown by the simulation example in Section 4 , with $\rho>1$ and $\lambda_{1}<1$ both sufficiently close to 1 , the mean values of the estimated parameters remain good approximations of the true values of these parameters.

\section{Simulation example}

In order to illustrate the results obtained in the previous paragraphs, we carry out simulations in which the feedforward transfer function of the equivalent loop has poles lying on the unit circle. The identification is achieved with the recursive output error with fixed compensator [Landau, Lozano, M’ Saad, Karimi, 2011] (p. 171). The transfer function to be identified is:

$$
G\left(z^{-1}\right)=\frac{B\left(z^{-1}\right)}{A\left(z^{-1}\right)}=\frac{0.05 z^{-1}-0.08 z^{-2}}{1-1.81 z^{-1}+z^{-2}}
$$

The two complex conjugate poles of which belong to the unit circle. At first we consider that no noise is added on the system output. For the identification purpose, the system is excited with a ten registers PRBS (with a one sample internal period). The a-posteriori predicted output $\widehat{y}(t)$ is given by: $\widehat{A}\left(t+1, q^{-1}\right) \widehat{y}(t+1)=\widehat{B}(t+$ $\left.1, q^{-1}\right) u(t+1)$ whereas the a-priori predicted output is: $\widehat{A}\left(t, q^{-1}\right) \widehat{y}^{o}(t+1)=\widehat{B}\left(t, q^{-1}\right) u(t+1)$. The output error algorithm with fixed compensator consists in computing the a-priori adaptation $\nu^{o}(t+1)$ according to:

$$
\nu^{o}(t+1)=\varepsilon^{o}(t+1)+L^{*}\left(q^{-1}\right) \varepsilon(t+1)
$$

where $\varepsilon(t+1)=y(t+1)-\widehat{y}(t+1)$ is the a-posteriori prediction error, $\varepsilon^{o}(t+1)=y(t+1)-\widehat{y}^{o}(t+1)$ is the a-priori prediction error, and $L^{*}\left(q^{-1}\right)=L\left(q^{-1}\right)-1$. Choosing $L\left(z^{-1}\right)=1-1.7195 z^{-1}+0.885 z^{-2}$, then according to [Landau, Lozano, M'Saad, Karimi, 2011], (pp. 160), the hyperstability theory imposes that the transfer function $L\left(z^{-1}\right) / A\left(z^{-1}\right)-\frac{\lambda_{2}}{2}$ be strictly real positive.

The PAA observation vector is given by:

$$
[-\widehat{y}(t)-\widehat{y}(t-1) u(t) u(t-1)]
$$

A first simulation consists in using the output error with fixed compensator, in which the forgetting factors are set to $\lambda_{1}=1, \lambda_{2}=1$ and $F(0)=I_{4}$. In this specific case, there is no chance that $L\left(z^{-1}\right) / A\left(z^{-1}\right)-\frac{\lambda_{2}}{2}$ be strictly real positive because of the zeros of $A\left(z^{-1}\right)$ on the unit circle. We check that the PAA does not converge: $\nu(t+1)$ does not tend towards zero, and the estimated parameters do not tend toward constants, as shown in Fig.3. The transfer function $L\left(\rho z^{-1}\right) / A\left(\rho z^{-1}\right)-\frac{\lambda_{2}}{2}$ be-
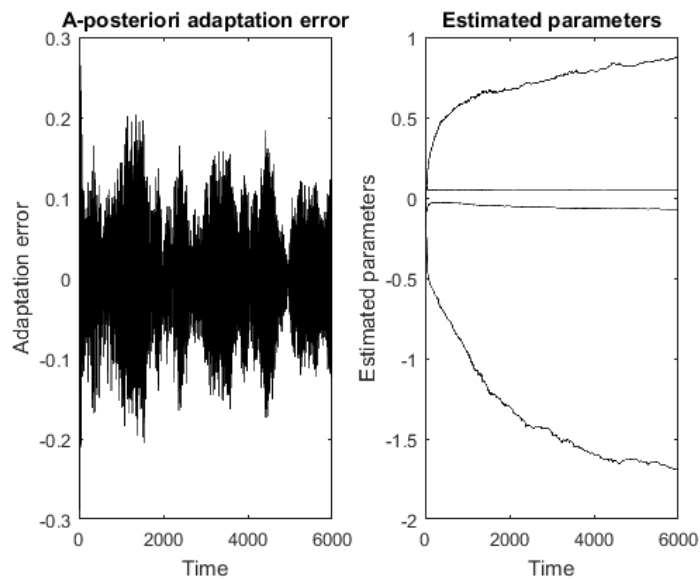

Fig. 3. Adaptation error and estimated parameters with $\lambda_{1}=1, \lambda_{2}=1$ (deterministic context)

comes strictly real positive for $\rho \approx 1.0025$. If we choose $\rho=1.0028$, and $\lambda_{1}=2-\rho^{2}=0.995$, we verify, as displayed in Fig.4, that identification under the same conditions entails the convergence of $\nu(t+1)$ towards 0 , and the estimated parameters to constant values: This is the result predicted by theorem 1 . The computed parameters mean relative errors from $t=2000$ to $t=6000$ are summarized in Table 1.

\begin{tabular}{|c|c|c|}
\hline Parameters & True Values & $\begin{array}{c}\text { Mean relative } \\
\text { errors }(\%)\end{array}$ \\
\hline$b_{1}$ & +0.05 & $2.210^{-2}$ \\
\hline$b_{2}$ & -0.08 & $1.010^{-2}$ \\
\hline$a_{1}$ & -1.81 & $8.210^{-6}$ \\
\hline$a_{2}$ & +1.00 & $5.210^{-4}$ \\
\hline
\end{tabular}

Table 1

True parameters value and mean relative errors (\%), from $t=2000$ to $t=6000$ (deterministic context). 

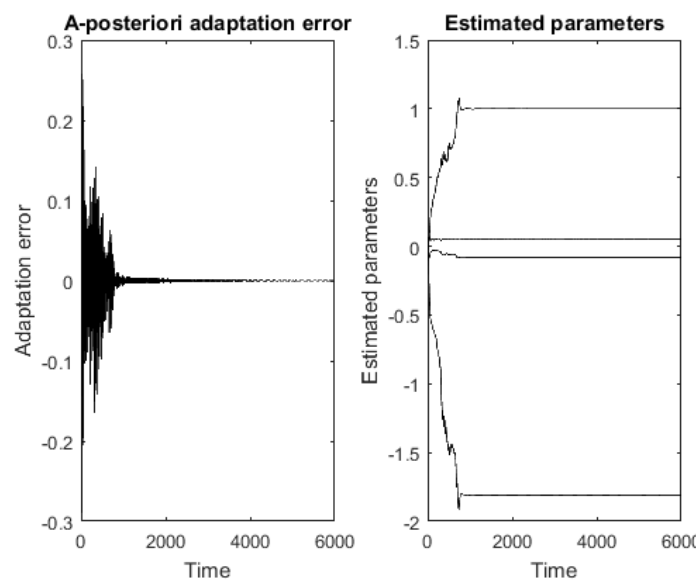

Fig. 4. Adaptation error and estimated parameters with $\lambda_{1}=0.995, \lambda_{2}=1$ (deterministic context)

We now add a pseudo-white Gaussian noise to the output $\mathrm{y}(\mathrm{t})$ with a signal/noise ratio equal to $10 \mathrm{~dB}$ (standard deviation). The Fig.5 shows that estimated parameters remain close to those obtained in a deterministic context, and that no divergence occurs because of the noise.
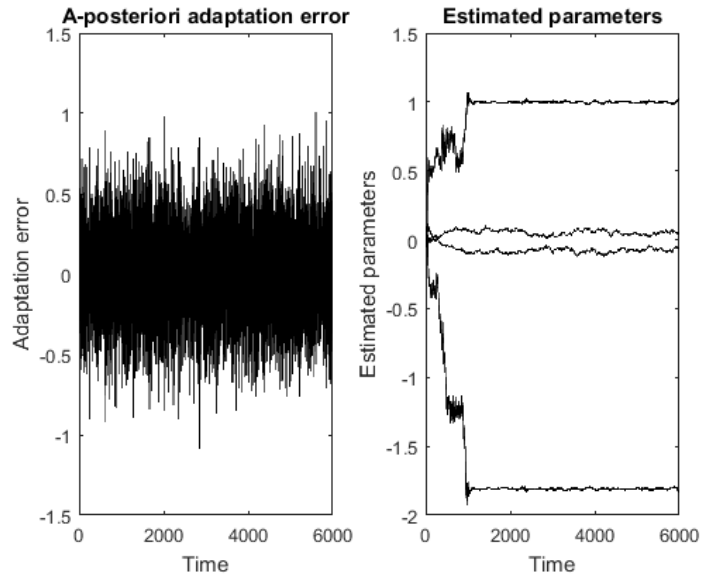

Fig. 5. Adaptation error and estimated parameters with $\lambda_{1}=0.995, \lambda_{2}=1$ (stochastic context)

The parameters mean relative errors from $t=2000$ to $\mathrm{t}=6000$, for a given noise realization, are displayed in Table 2 .

\begin{tabular}{|c|c|c|}
\hline Parameters & True Values & $\begin{array}{c}\text { Mean relative } \\
\text { errors }(\%)\end{array}$ \\
\hline$b_{1}$ & +0.05 & 2.5 \\
\hline$b_{2}$ & -0.08 & 2.3 \\
\hline$a_{1}$ & -1.81 & $5.010^{-2}$ \\
\hline$a_{2}$ & +1.00 & $1.210^{-1}$ \\
\hline
\end{tabular}

Table 2

True parameters value and mean relative errors (\%), from $t=2000$ to $t=6000$ (stochastic context).

\section{Conclusion}

We have shown how the recursive parameter estimation algorithm can be used in cases where the classical way of doing (with the forgetting factors set to 1) provokes divergence. Our method is based on two convergence theorems which are generalizations of those existing. So, convergence can be guaranteed even when the transfer function associated with the equivalent closed-loop does not satisfy the classical real positiveness condition involved in hyperstability theory. These theorems address both the deterministic and the stochastic context, and are particularly well suited to identify systems which may have poles on the unit circle.

\section{References}

[Anderson, Moore, 1971] Anderson B.D.O, Moore J.B. (1971). Optimal Control- Linear quadratic method., Saddle River NJ: Prentice-Hall.

[Anderson, Moore 1979] Anderson B.D.O, Moore J.B. (1979). Optimal filtering. Saddle River NJ: Prentice Hall.

[Bourlès, 1987] Bourlès H. (1987). Alpha-stability and robustness of large-scale interconnected systems. International journal of control 45(6), 2221-2232.

[Bourlès, 1990] Bourlès H, Joannic Y, Mercier O. (1990). Rhostability and robustness : Discrete-time case. International journal of control 52(5), 1217-1239

[Landau, 1965] Landau I.D (1965). A hyperstability criterion for model reference adaptive control, systems. IEEE Transactions on automatic control 14, 552-555.

[Landau, Silveira, 1979] Landau I.D., Silveira H.M. (1979). A stability theorem with applications to adaptive control. IEEE Transactions on automatic control 24(2), 305-312.

[Landau, 1982] Landau I.D. (1982). Near supermartingales for convergence analysis of recursive identification and adaptive control schemes. International journal of control 35(2), 197226 .

[Landau, Lozano, M' Saad, Karimi, 2011] Landau I.D, Lozano R, M' Saad M, Karimi A. (2011). Adaptive control (second edition). London: Springer Verlag.

[Lozano, 1983] Lozano R. (1983). Convergence analysis of recursive identification algorithm with forgetting factor, Automatica 19(1), 95-97. 